\title{
Cocaine-induced Psychosis and Brain-derived Neurothrophic Factor in Patients with Cocaine Dependence: Report of Two Cases
}

\author{
Carlos Roncero ${ }^{1,2,3}$, Raul Felipe Palma-Álvarez ${ }^{2}$, Elena Ros-Cucurull, ${ }^{1,2,3}$, Carmen Barral ${ }^{1,2}$, Begoña Gonzalvo², \\ Margarida Corominas-Roso, Miguel Casas ${ }^{2,3}$, Lara Grau-López ${ }^{1,2,3}$ \\ ${ }^{1}$ Addictions and Dual Diagnosis Unit, Vall d'Hebron University Hospital-Public Health Agency of Barcelona (ASPB), Centro de Investigación \\ Biomédica en Red de Salud Mental (CIBERSAM), ${ }^{2}$ Department of Psychiatry, Vall d'Hebron University Hospital, CIBERSAM, ${ }^{3}$ Department \\ of Psychiatry and Legal Medicine, Universitat Autònoma de Barcelona, Barcelona, Spain
}

\begin{abstract}
Brain-derived neurotrophic factor (BDNF) is linked to numerous brain functions. In addition, BDNF alterations contribute to neurological, mental, and addictive disorders. Cocaine dependence has received much attention recently due to its prevalence and psychological effects. Symptoms of psychosis are one of the most serious adverse events precipitated by cocaine use. It is particularly important to identify patients at risk of developing cocaine-induced psychosis (CIP). We described two cases of patients with cocaine dependence who presented with CIP and had changes in their BDNF levels during the psychotic episode. BDNF levels were initially low in both patients, and then decreased by more than $50 \%$ in association with CIP. The relationship between BDNF and psychosis is described in the literature. These cases revealed that BDNF levels decreased during a CIP episode and, thus, it is necessary to investigate BDNF and its relationship with CIP further.
\end{abstract}

KEY WORDS: Cocaine-induced psychosis; Cocaine dependence; Brain-derived neurotrophic factor; Biological marker; Psychosis.

\section{INTRODUCTION}

Brain-derived neurotrophic factor (BDNF) is a neurotrophin involved in neurodevelopment, neuroprotection, synapse regulation, and learning and memory. ${ }^{1-4)}$ BDNF interacts with dopamine, glutamine, and other neurotransmitter systems. ${ }^{1,5)}$ Because of those interactions, BDNF has also been proposed as an illness and prognostic marker for several neurologic, mental, and addictive disorders. ${ }^{2-4,6,7)}$ Many studies have indicated a correlation between schizophrenia and psychotic episodes with decreased BDNF levels (including two meta-analyses) ${ }^{1,8-11)}$; this relationship is probably mediated by alterations in synaptic function and connectivity. ${ }^{9}$ It is known that BDNF is decreased during a patient's first psychotic episode ${ }^{10,11)}$ this decrease becomes more marked with disease progression. ${ }^{8)}$ Additionally, an age-related reduction in BDNF has been observed in blood. ${ }^{9)}$ Antipsychotics

\footnotetext{
Received: June 23, 2015 / Revised: July 31, 2015

Accepted: August 17, 2015

Address for correspondence: Carlos Roncero, MD, PhD Department of Psychiatry, Vall d'Hebron University Hospital, Passeig Vall d'Hebron, 119-129, 08035 Barcelona, Spain Tel: +34-93-489-42-95, Fax: +34-93-489-45-87 E-mail: croncero@vhebron.net
}

and antidepressants can affect BDNF levels; however, findings regarding the effects of antipsychotics are equivocal. Some investigations showed that BDNF levels differed following treatment with typical versus atypical antipsychotics. ${ }^{2)}$ Evidence suggests that typical antipsychotics decrease BDNF levels, but mixed results have been found regarding atypical antipsychotics. ${ }^{2)}$

BDNF has recently been associated with cocaine-induced psychosis (CIP). The term CIP is used to describe symptoms of psychosis (suspiciousness, paranoia, delusions, and hallucinations) that are precipitated by cocaine use. ${ }^{5,12)}$ For this reason, BDNF levels could act as a biological marker of psychosis in patients with cocaine dependence. Psychotic symptoms have been reported in more than $50 \%$ of patients with cocaine dependence; this percentage ranges from $48 \%$ to $88 \%$ depending on the study variables. ${ }^{13)}$

Numerous risk factors are associated with $\mathrm{CIP}^{3,12,13)}$ including age at first use, amount of substance, route of administration, use of other substances, personal vulnerability, and comorbid psychiatric disorders. ${ }^{12,13)}$ Interestingly, some studies of patients with chronic cocaine addiction also reported low levels of BDNF.") However, there is

(c) This is an Open-Access article distributed under the terms of the Creative Commons Attribution Non-Commercial License (http://creativecommons.org/licenses/by-nc/4.0) which permits unrestricted non-commercial use, distribution, and reproduction in any medium, provided the original work is properly cited. 
no consensus regarding the role of BDNF level on cocaine use relapse. $^{14)}$ There are few studies of the relationship between BDNF and CIP. To the best of our knowledge, only one study investigated patients with CIP and their BDNF levels. That study concluded that BDNF could play a role in psychotic symptoms in cocaine users because BDNF levels did not rise after withdrawal in patients with CIP compared with patients without a history of CIP. ${ }^{5)}$ This conclusion was reached following an observed correlation between a decreased BDNF level and CIP, which is similar to findings in patients with schizophrenia and other psychosis. ${ }^{5)}$ In this context, BDNF and other biological markers might be useful in identifying patients with a vulnerability for CIP and may also be a prognostic factor. ${ }^{5)}$

Not only do neuropsychiatric diseases alter BDNF levels, but many other conditions such as diet, exercise, nicotine, and caffeine use alter BDNF levels as well. Some research suggests that diet and exercise could interact with BDNF expression. ${ }^{15,16)}$ Caffeine increased BDNF expression in some animal models, ${ }^{17,18)}$ as did nicotine ${ }^{19)}$; however, not all investigations have come to this conclusion. $^{19)}$

We present two patients with cocaine dependence and CIP who were evaluated and included in an independent clinical trial conducted by the Psychiatry Department at the Vall d'Hebron University Hospital (Barcelona, Spain). These patients were hospitalized for cocaine detoxification, and serum BDNF levels were measured at the beginning of hospitalization as well as 12 days later. Both patients presented with psychotic symptoms during hospitalization, and were thus excluded from the clinical trial. Interestingly, BDNF levels further decreased during the psychotic episode compared with the levels found upon initial measurement.

\section{CASE}

The two patients were participants of a clinical trial that was conducted by the Psychiatry Department at the Vall d'Hebron University Hospital in which a combination of caffeine, biperiden, and placebo was evaluated as a treatment for cocaine dependence (www.clinicaltrials.gov identifier: NCT00495092). That trial was approved by The Clinical Research Ethics Committee of the Vall d'Hebron University Hospital. All patients signed an informed consent form and agreed to be included in the study. Both individuals described earlier were excluded from that trial because they presented with psychotic symptoms during the study.
During their hospital stay, the patients were in a locked unit with no substance access and restrictions on visitation. They were detoxicated, and drug testing was performed during their hospitalization. The patients were seen every day by the psychiatrist who performed their initial clinical assessment and diagnosis (according the Diagnostic and Statistical Manual of Mental Disorders, fourth edition [DSM-IV] criteria). As part of the original trial, they received caffeine or placebo; the general conditions were the same for all inpatients (e.g., diet, exercise).

As per the protocol, two blood samples were collected from each patient in the original trial: one at the beginning and the other 12 days after the first sample was taken. No other samples were collected for BDNF assay. Serum BDNF was determined by sampling $8 \mathrm{ml}$ of peripheral blood between 10:00 am and 12:00 am to avoid circadian interference. The samples were kept in anticoagulant-free tubes for 2 hours at $4^{\circ} \mathrm{C}$. Serum BDNF was measured using a sandwich enzyme-linked immunosorbent (ELISA) system for quantitative protein measurement (Aushon Biosystems, Billerica, MA, USA).

\section{Case 1}

A 25-year-old male came to the detoxification unit to participate in the clinical trial. His last cocaine use was 24 hours prior to hospitalization. He presented with simple reference delusions with behavioral repercussion; furthermore, he had auditory hallucinations and some simple visual hallucinations. This psychotic pattern developed on day 5 after unit admission, and the episode duration was approximately 4 days. The patient did not have other mental nor neurological symptoms (e.g., his consciousness was never compromised, and no attention deficits were observed). This episode was investigated, and no metabolic etiology was established. A benzodiazepine was prescribed. On the first day of hospitalization, his initial BDNF level was measured: the BDNF level was low $(70.94 \mathrm{ng} / \mathrm{ml})$, and this level decreased further 12 days later $(49.05 \mathrm{ng} / \mathrm{ml})$.

The patient's psychiatric history included a major depressive (MD) episode, diagnosed 6 years prior, with total recovery. Cocaine and nicotine dependence were diagnosed before his hospitalization. He also had alcohol and benzodiazepine dependence, but had not recently used those substances. He sporadically used cannabis during his youth. No other substances were used. The patients did not have any other mental or medical antecedents, or antecedents of psychotic episodes. 


\section{Case 2}

A 52-year-old male presented with a psychotic episode two days after hospitalization. This episode was characterized by suspiciousness, partially structured reference and prejudice delusions, and disorganized behavior. $\mathrm{He}$ had a short time and spatial disorientation at the beginning of this state, followed by normal attention and consciousness. Toxin screening, blood studies, and brain tomography were performed to rule out an organic cause for the psychosis. BDNF levels were measured on the first day of hospitalization $(52.82 \mathrm{ng} / \mathrm{ml})$ and again 12 days later $(29.93 \mathrm{ng} / \mathrm{ml})$ as per the protocol. The initial BDNF level was low; the second measurement was lower than the initial measurement and coincided with the patient's psychotic symptoms. The psychotic episode was treated with $200 \mathrm{mg}$ /day quetiapine, and the psychotic symptoms were improved 10 days later.

No relevant antecedents were noted in his medical history (only surgical intervention for an anal abscess). However, the patient's psychiatric history included the previous diagnosis of a cluster A personality disorder (PD), not specified. He had a long toxicological history; he only had cocaine and nicotine dependence upon clinical hospitalization. He previously had heroin, cannabis, and alcohol dependence. There was no antecedent of psychotic episodes (with or without substance use).

\section{DISCUSSION}

Here we reported two cases with cocaine dependence who had initial low serum BDNF levels upon hospitalization that decreased further in association with psychotic symptoms. Upon hospitalization, the patients were diagnosed with cocaine and tobacco dependence; no previous psychotic episodes (with or without substance use) were documented. It is important to point out that other causes of psychosis were ruled out in both patients (organic or pharmacological etiologies). It was important to consider the psychiatric history of both patients. In Case 1 , the patient had a previous episode of MD, which must be considered when interpreting the BDNF level data. MD disorder has been correlated with low BDNF levels, which were then enhanced after antidepressant treatment. ${ }^{20)}$ In this patient, MD and psychotic episodes may have represented nervous system damage mediated by BDNF system alterations. In Case 2, a diagnosis of cluster A PD, not specified, was significant because evidence exists suggesting that PD could be a risk factor for psychosis conversion in at-risk patients. ${ }^{21)}$ There are few studies on the relationship between PD and BDNF. No studies exist on cluster A PD and BDNF; however, epigenetic changes in BDNF expression (DNA methylation) were investigated in patients with borderline $\mathrm{PD},{ }^{22)}$ and one study revealed a higher BDNF plasma level in patients with borderline PD. ${ }^{22)}$ Another study reported that platelet BDNF was decreased in males with borderline PD. ${ }^{23)}$

The cases that we presented are in line with evidence suggesting that BDNF may be useful as a marker not only of psychotic episodes or schizophrenia ${ }^{8,9)}$ but also of CIP ${ }^{5)}$ and addictive disorders. ${ }^{3,5)}$ Our cases presented with an initial low level of BDNF similar to other reports of patients with cocaine dependence ${ }^{3)}$ and schizophrenia. ${ }^{6,8,9,24)}$ Some researchers reported that the initial low levels increase until they normalize during cocaine withdrawal in patients without a history of $\mathrm{CIP}^{3,5)}$ Furthermore, in patients who use crack cocaine, BDNF levels increase after crack cocaine withdrawal. ${ }^{25)}$ We did not observe an increase in BDNF levels following cocaine withdrawal in our cases; conversely, we observed a marked reduction of those levels in our patients that coincided with psychotic episodes. BDNF reportedly decreased during the first psychotic episode, ${ }^{10)}$ and was associated with the duration of the first psychotic episode. ${ }^{11)}$ These data are interesting because few studies measured BDNF levels during a patient's first psychotic episode, and many studies were conducted in patients with chronic psychosis. ${ }^{9)}$ Employing BDNF as a biological marker could be useful in some aspects of psychosis, with the best use potentially being as a prognostic or disease activity biomarker for psychotic episodes. ${ }^{1,5,8-11)}$ Additionally, BDNF has been studied as a marker for different aspects of drug addictions (initiation, maintenance, abstinence, craving, risk factors) and also as a predictive factor for cocaine relapse. ${ }^{1424)}$ The interaction between BDNF and the dopaminergic system, particularly in midbrain dopaminergic neurons, could explain its relationship with drug reward and relapse. ${ }^{1,3,24)}$

Finally, there are some limitations to extrapolating these cases. First, patients received placebo, caffeine, or biperiden during the original trial. In animal models, caffeine increased BDNF expression. However, the BDNF levels did not differ among the groups (including the placebo group) in the original trial. ${ }^{3,5)}$ Both patients had nicotine dependence. Many animal and human studies found that nicotine increased BDNF expression and its levels ${ }^{19}$; however, some findings were inconsistent. ${ }^{19)}$ Specifically, one study showed that schizophrenic smokers had significantly increased BDNF levels and fewer positive symptoms than nonsmokers. ${ }^{19)}$ In our cases, BDNF levels 
were decreased, even with nicotine use. Both patients had the same exercise and diet conditions, which is important because diet and exercise can modify BDNF expression. ${ }^{15,16}$

$\mathrm{BDNF}$ is an important neuropsychiatric biomarker that should be investigated further; its biological functions could explain its important correlation with several diseases. The correlation between BDNF and many aspects of psychotic episodes (duration, diagnosis, prognosis, etc.) could make it useful as a biological marker. The cases reported herein suggest a correlation between BDNF decrease and CIP. Although we only reported two cases, and other factors might be related to changes in BDNF levels, this information could be helpful in order to elucidate whether BDNF could be a biological marker for CIP. Future research should be conducted in this area, including specific trials that compared cocaine effects on BDNF levels in patients with CIP.

\section{Acknowledgments}

We are grateful to Marta Ribasés, Cristina Sánchez-Mora and Laia Rodriguez-Cintas for their collaboration and support.

The initial study was supported by the Grant from the Subdirecció General de Drogodependències to study cocaine dependence, a Grant from the Instituto Carlos III: FIS PI13/1911 "Alucinaciones cenestésicas: factor pronóstico en dependientes de cocaína" and Grant from the Delegación del Gobierno para el Plan Nacional sobre Drogas 2013I044: "Complicaciones clínicas y accidentabilidad asociadas a la presencia de síntomas psicóticos en consumidores de cocaína". The PND and FIS had no further role in study design (collection, analysis, interpretation of data, writing of the report, and in the decision to submit the paper for publication).

\section{REFERENCES}

1. Nurjono M, Lee J, Chong SA. A review of brain-derived neurotrophic factor as a candidate biomarker in schizophrenia. Clin Psychopharmacol Neurosci 2012;10:61-70.

2. Nieto R, Kukuljan M, Silva H. BDNF and schizophrenia: from neurodevelopment to neuronal plasticity, learning, and memory. Front Psychiatry 2013;4:45.

3. Corominas-Roso M, Roncero C, Eiroa-Orosa FJ, Gonzalvo B, Grau-Lopez L, Ribases M, et al. Brain-derived neurotrophic factor serum levels in cocaine-dependent patients during early abstinence. Eur Neuropsychopharmacol 2013; 23:1078-1084.

4. Corominas M, Roncero C, Ribases M, Castells X, Casas M. Brain-derived neurotrophic factor and its intracellular signaling pathways in cocaine addiction. Neuropsychobiology 2007; 55:2-13.

5. Corominas-Roso M, Roncero C, Eiroa-Orosa FJ, Ribasés M, Barral C, Daigre C, et al. Serum brain-derived neurotrophic factor levels and cocaine-induced transient psychotic symptoms. Neuropsychobiology 2013;68:146-155.

6. Autry AE, Monteggia LM. Brain-derived neurotrophic factor and neuropsychiatric disorders. Pharmacol Rev 2012; 64:238-258.

7. Zhang H, Ozbay F, Lappalainen J, Kranzler HR, van Dyck $\mathrm{CH}$, Charney DS, et al. Brain derived neurotrophic factor (BDNF) gene variants and Alzheimer's disease, affective disorders, posttraumatic stress disorder, schizophrenia, and substance dependence. Am J Med Genet B Neuropsychiatr Genet 2006;141B:387-393.

8. Fernandes BS, Steiner J, Berk M, Molendijk ML, Gonzalez-Pinto A, Turck CW, et al. Peripheral brain-derived neurotrophic factor in schizophrenia and the role of antipsychotics: meta-analysis and implications. Mol Psychiatry 2015;20:1108-1119.

9. Green MJ, Matheson SL, Shepherd A, Weickert CS, Carr VJ. Brain-derived neurotrophic factor levels in schizophrenia: a systematic review with meta-analysis. Mol Psychiatry 2011;16:960-972.

10. Toll A, Mané A. Brain-derived neurotrophic factor levels in first episode of psychosis: A systematic review. World $J$ Psychiatry 2015;5:154-159.

11. Rizos EN, Michalopoulou PG, Siafakas N, Stefanis N, Douzenis A, Rontos I, et al. Association of serum brainderived neurotrophic factor and duration of untreated psychosis in first-episode patients with schizophrenia. Neuropsychobiology 2010;62:87-90.

12. Roncero C, Daigre C, Gonzalvo B, Valero S, Castells X, Grau-López L, et al. Risk factors for cocaine-induced psychosis in cocaine-dependent patients. Eur Psychiatry 2013;28: 141-146.

13. Roncero C, Ros-Cucurull E, Daigre C, Casas M. Prevalence and risk factors of psychotic symptoms in cocaine-dependent patients. Actas Esp Psiquiatr 2012;40:187-197.

14. D'Sa C, Fox HC, Hong AK, Dileone RJ, Sinha R. Increased serum brain-derived neurotrophic factor is predictive of cocaine relapse outcomes: a prospective study. Biol Psychiatry 2011;70:706-711.

15. Godar R, Dai Y, Bainter H, Billington C, Kotz CM, Wang CF. Reduction of high-fat diet-induced obesity after chronic administration of brain-derived neurotrophic factor in the hypothalamic ventromedial nucleus. Neuroscience 2011; 194:36-52.

16. Zoladz JA, Pilc A. The effect of physical activity on the brain derived neurotrophic factor: from animal to human studies. J Physiol Pharmacol 2010;61:533-541.

17. Ardais AP, Borges MF, Rocha AS, Sallaberry C, Cunha RA, Porciúncula LO. Caffeine triggers behavioral and neurochemical alterations in adolescent rats. Neuroscience 2014; 270:27-39.

18. Capiotti KM, Menezes FP, Nazario LR, Pohlmann JB, de Oliveira GM, Fazenda L, et al. Early exposure to caffeine affects gene expression of adenosine receptors, DARPP-32 and BDNF without affecting sensibility and morphology of developing zebrafish (Danio rerio). Neurotoxicol Teratol 2011;33:680-685.

19. Zhang XY, Xiu MH, Chen da C, Yang FD, Wu GY, Lu L, et al. Nicotine dependence and serum BDNF levels in male patients with schizophrenia. Psychopharmacology (Berl) 2010;212:301-307.

20. Molendijk ML, Spinhoven P, Polak M, Bus BA, Penninx BW, Elzinga BM. Serum BDNF concentrations as peripheral manifestations of depression: evidence from a systematic review and meta-analyses on 179 associations $(N=9484)$. 
Mol Psychiatry 2014;19:791-800.

21. Schultze-Lutter F, Klosterkötter J, Michel C, Winkler K, Ruhrmann S. Personality disorders and accentuations in at-risk persons with and without conversion to first-episode psychosis. Early Interv Psychiatry 2012;6:389-398.

22. Perroud N, Salzmann A, Prada P, Nicastro R, Hoeppli ME, Furrer $\mathrm{S}$, et al. Response to psychotherapy in borderline personality disorder and methylation status of the BDNF gene. Transl Psychiatry 2013;3:e207.

23. Koenigsberg HW, Yuan P, Diaz GA, Guerreri S, Dorantes $\mathrm{C}$, Mayson S, et al. Platelet protein kinase $C$ and brain- derived neurotrophic factor levels in borderline personality disorder patients. Psychiatry Res 2012;199:92-97.

24. Balaratnasingam S, Janca A. Brain derived neurotrophic factor: a novel neurotrophin involved in psychiatric and neurological disorders. Pharmacol Ther 2012;134:116-124. 25. von Diemen L, Kapczinski F, Sordi AO, de Magalhães Narvaez JC, Guimarães LS, Kessler FH, et al. Increase in brain-derived neurotrophic factor expression in early crack cocaine withdrawal. Int J Neuropsychopharmacol 2014;17: $33-40$. 